\title{
An approach for considering ionization of light atoms by relativistic projectiles generating strong electromagnetic fields
}

\author{
A.B.Voitkiv ${ }^{1,2}$, B.Najjari ${ }^{2}$ and J.Ullrich ${ }^{2}$ \\ ${ }^{1}$ Institute for Theoretical Physics, University of Giessen, Heinrich-Buff-Ring 16, D-35392 \\ Giessen, Germany \\ ${ }^{2}$ Max-Planck-Institut für Kernphysik, Saupfercheckweg 1, D-69117, Heidelberg, Germany
}

(April 9, 2003)

\begin{abstract}
We propose an approach to treat ionization of light atoms in relativistic collisions with highly charged ions where the electromagnetic field generated by the ions can be very strong. The approach is based on the observation, that, for the collision with a certain momentum transfer, either the relativistic effects, connected with the collision velocity approaching the speed of light, or the higher order terms in the corresponding Born series in the projectile-target interaction can be of importance for the ionization process. The approach consists of dividing all collisions into those with 'small' and 'large' momentum transfers which are described by (the first order of) the relativistic Born and the Glauber approximations, respectively. The approach is applied to describe helium single ionization by $1 \mathrm{GeV} / \mathrm{u} \mathrm{U}^{92+}$ projectiles.
\end{abstract}

PACS:34.10.+x, 34.50.-s, 34.50.Fa 


\section{INTRODUCTION}

The study of different aspects of relativistic ion-atom collisions has attracted much attention during the last two decades (for a review see [1]- [4]). Among various processes, target ionization by charged point-like projectiles moving at velocities approaching the speed of light was addressed in numerous publications which were mainly concerned with ionization of the $K$-shell of very heavy atoms. The electromagnetic field of ions, which move at velocities approaching the speed of light, substantially differs from that produced by nonrelativistic charged projectiles. This may result in crucial changes in atomic cross sections compared to what one would expect on the basis of a nonrelativistic consideration.

Relatively less attention has been paid to the study of collisions of relativistic projectiles with light atoms, in particular, to ionization of such atoms in the collisions (see [5]- [12]). However, compared to the ionization of the $K$ shell of heavy elements, the latter process has interesting features which make it worth of more extensive studies. For example, the motion of the main bulk of electrons emitted from light atoms in such collisions is still far from being relativistic. Therefore, one faces an interesting interplay between the relativistic relative projectile-target motion and the basically nonrelativistic dynamics of the target electrons. In addition, the low-energy character of the electron emission permits one to use sophisticated experimental methods for performing kinematically complete measurements for the reaction products. The last but not least important feature we would like to mention here takes place when relativistic highly charged ions are involved. Such ions may represent an effectively strong perturbation for light targets (or for outer-shell electrons of heavy targets) even in collisions at very high energies. The latter will especially be the case if multi-electron transitions occurs in the target. For example, it is known for more than a decade that relativistic highly charged ions are capable of producing emission from atomic outer shells where several electrons are removed in a single collision with a rather high probability [13]. Therefore, there is, in general, the need to combine in a theoretical treatment of ionization of light targets the description of relativistic effects, associated with collision velocity approaching the speed of light, with those arising from the exchange of many (virtual) photons between the colliding projectile and target.

Very recently we have addressed a few aspects of ionization of light targets by relativistic projectiles in [14]- [16] where the considerations were based on the first order of the relativistic Born approximation. Within this approximation a proper account may be taken of the relativistic effects but the projectile-target interaction is effectively reduced to the exchange of just a single photon. It is the intention of the present paper to attempt to propose such an approach for calculating various cross sections including the fully differential ones, which allows one to take (approximately) into account the higher order terms in the projectiletarget interaction (the so called many-photon exchange). Our attention will be restricted to emission of electrons with velocities much smaller than that of a projectile which are nonrelativistic in the final state and form the main bulk of the ejected electrons.

The paper is organized as follows. The approach is described in the Section II. In the Section III it is applied to helium single ionization by $1 \mathrm{GeV} / \mathrm{u} \mathrm{U}^{92+}$ ions.

Atomic units are used throughout except where otherwise stated. 


\section{GENERAL CONSIDERATION}

Let us consider the collision between an atom and a point-like bare ion with a charge $Z_{p}$. The atom is a light atom with the nuclear charge $Z_{T} \ll c$, where $c=137$ is the speed of light. The atom is initially in an internal state $\psi_{0}\left(\boldsymbol{\tau}_{N_{T}}\right)$ with an energy $\varepsilon_{0}$, where $\boldsymbol{\tau}_{N_{T}}=\left\{\mathbf{r}_{1}, \mathbf{r}_{2}, \ldots, \mathbf{r}_{N_{T}}\right\}$ are the coordinates of the $N_{T}$ atomic electrons with respect to the atomic nucleus. It is convenient to take a reference frame where the atom (target) is initially at rest. In this frame the ion (projectile) initially moves with a relativistic velocity $\mathbf{v}_{p}$. As a result of the collision the projectile changes its momentum from $\mathbf{P}_{i}$ to $\mathbf{P}_{f}=\mathbf{P}_{i}-\mathbf{q}$. The momentum $\mathbf{q}$ is transferred to the target which makes a transition into its final (internal) state $\psi_{n}\left(\boldsymbol{\tau}_{N_{T}}\right)$ with an energy $\varepsilon_{n}$.

There are two important points which one should take into account. First, excluding the negligibly small part of the electron emission from the target represented by very high energy electrons which will not be considered here, the motion of the target electrons in the process of relativistic collision remains to be nonrelativistic in the target frame. Therefore, these electrons can be described by nonrelativistic wavefunctions. Second, since exact (in the nonrelativistic sense) wavefunctions are not available for targets with two and more electrons, the Coulomb gauge should be used to describe the field of a relativistic projectile $[15]$.

1. Let us start with considering the projectile-target collisions within the first order of the relativistic Born approximation. This can be done in a standard way by dealing with the first order Feynmann graph (see e.g. [4], [16] and references therein). Taking into account the two points mentioned in the previous paragraph the cross section, differential in the momentum transfer $\mathbf{q}$, of a process where the target makes the transition $\psi_{0} \rightarrow \psi_{n}$ can be written as [15]- [16]

$$
\frac{d \sigma_{0 \rightarrow n}}{d \mathbf{q}_{\perp}}=\frac{4 Z_{p}^{2}}{v_{p}^{2}}\left|<\psi_{n}\left(\boldsymbol{\tau}_{N_{T}}\right)\right| \hat{T}_{1 B}\left|\psi_{0}\left(\boldsymbol{\tau}_{N_{T}}\right)>\right|^{2} .
$$

In Eq.(1) $\psi_{0, n}$ are the many-electron wavefunctions which are solutions of the nonrelativistic Schrödinger equation for the free target

$$
H_{a t}=\sum_{j}\left(-\frac{\Delta_{j}}{2}-\frac{Z_{t}}{r_{j}}\right)+\sum_{i>j} \frac{1}{r_{j i}}
$$

where $r_{i j}$ are the distances between the $\mathrm{i}$-th and $\mathrm{j}$-th atomic electrons.

The transition operator $\hat{T}_{1 B}$ in (1) is given by [15]- [16]

$$
\hat{T}_{1 B}=\frac{1}{\mathbf{q}_{\perp}^{2}+q_{m i n}^{2}} \sum_{i=1}^{N_{T}} \exp \left(i \mathbf{q} \cdot \mathbf{r}_{i}\right)-\frac{1}{\mathbf{q}_{\perp}^{2}+q_{m i n}^{2}-\frac{\omega_{n 0}^{2}}{c^{2}}} \sum_{i=1}^{N_{T}} \exp \left(i \mathbf{q} \cdot \mathbf{r}_{i}\right) \mathbf{g} \cdot \hat{\mathbf{p}}_{i}
$$

In Eq.(3) the polarization vector $\mathbf{g}=\frac{1}{c^{2}}\left(\mathbf{v}_{p}-\frac{\omega_{n 0}}{q^{2}} \mathbf{q}\right)$ is perpendicular to the momentum transfer, $\mathbf{g} \cdot \mathbf{q}=0, \hat{\mathbf{p}}_{i}$ is the electron momentum operator for the $i$-th target electron, $\gamma=1 / \sqrt{1-v_{p}^{2} / c^{2}}$ is the collision Lorentz factor. The sums in (3) run over all target electrons. 
It is understood in (1)-(3) that the $z$-axis is directed along the initial projectile velocity: $\mathbf{v}_{p}=\left(0,0, v_{p}\right)$. The momentum transfer is written as $\mathbf{q}=\left(\mathbf{q}_{\perp}, q_{\min }\right)$, where the twodimensional part $\mathbf{q}_{\perp}$ of the momentum transfer is perpendicular to the $z$-axis and the $z$ component of the momentum transfer is given by $q_{\text {min }}=\frac{\omega_{n 0}}{v_{p}}$ with $\omega_{n 0}=\varepsilon_{n}-\varepsilon_{0}$ being the target transition frequency. Since $\omega_{n 0} \sim Z_{t}^{2}$, one can estimate $q_{\min } \sim Z_{t}^{2} / v_{p}$.

The transition operator (3) consists of two terms. The first term is the contribution to the cross section originating from the projectile scalar potential which in the Coulomb gauge is just the unretarded (nonrelativistic) Coulomb potential. This term is pretty the same as in nonrelativistic collisions ${ }^{1}$. The relativistic effects, therefore, have to be contained in the second term of (3) which is due to the vector potential of the projectile field. These effects can be subdivided into: i) the additional contribution to the projectile's electric field arising from the vector potential; ii) the retardation, described by the term $\omega_{n 0}^{2} / c^{2}$ in $(3)$, which makes the above mentioned contribution to the electric field much more important than it would be in the absence of $\omega_{n 0}^{2} / c^{2}$; and iii) the projectile's magnetic field, which is just of minor importance for the case of ionization of light targets.

It is known (see [14]) that the second term strongly influences the transition amplitudes only if the transverse momentum component is small enough, $q_{\perp} \lesssim q_{\text {min }}$. When $q_{\perp}$ increases the relative importance of the second term decreases and for $q_{\perp} \gg q_{\min }$ its contribution to the target ionization becomes already negligible [14].

It is important to note that the simple analysis, based on the so-called light-cone approximation [17] (see also [18] and [19]), clearly shows that the conclusion about the unimportance of the relativistic effects for collisions with $q_{\perp} \gg q_{\text {min }}$ is not just a peculiarity of the first order consideration but remains when the higher order terms in the projectile-target interaction are taken into account.

Thus, keeping in mind that $q_{\min } \sim Z_{t}^{2} / v_{p}$ one can conclude that the relativistic effects will be of great and minor importance for collisions with $q_{\perp} \lesssim Z_{t}^{2} / v_{p}$ and $q_{\perp} \gg Z_{t}^{2} / v_{p}$ (but $\left.q_{\perp} \ll c\right)$, respectively.

2. On the other hand, the importance of the many-photon exchange between the projectile and the target is expected to increase with increasing the transverse momentum transfer $q_{\perp}$ since collisions with larger $q_{\perp}$ in general correspond to collisions with smaller impact parameters where the interaction is stronger. The analysis, based on the Glauber approximation which is known to be a good approximation for treating high-energy collisions with $q_{\perp} \gg q_{\min }$ (see also 5.), shows that at $q_{\perp} \ll \lambda Z_{t}$ (where the quantity $\lambda$ depends on the projectile charge and velocity and is roughly proportional to $\frac{v_{p}}{Z_{p}}$ ) the exchange of more than one photon still plays a minor role. The same analysis also indicates that with increasing $q_{\perp}$ the importance of the multiphoton exchange increases and that at $q_{\perp} \lesssim Z_{t}$ these processes may already strongly influence the emission process.

\footnotetext{
${ }^{1}$ In eqs.(1)-(3) relativistic collision kinematics has been taken into account. However, since the emitted electrons are nonrelativistic and the relativistic projectile undergoes just a very small deflection in the collision, the kinematics of the target nucleus and target electrons remains as if the collision were nonrelativistic.
} 
Now, by combining the inequality $q_{\perp} \gg Z_{t}^{2} / v_{p}$ with $q_{\perp} \ll \lambda Z_{t}$ we see that there may exist a range of momentum transfers, defined as $Z_{t} \frac{Z_{t}}{v_{p}} \ll q_{\perp} \ll \lambda Z_{t}$, where both the relativistic effects and the influence of the many-photon exchange are weak.

3. Summarizing the considerations of the subsections $\mathbf{1}$ and $\mathbf{2}$ we see that one can distinguish three different ranges of momentum transfers. i) The range of 'small' momentum transfers $q_{\perp} \lesssim q_{\text {min }}$, where the relativistic effects may be important. ii) The range of 'large' momentum transfers which begins, roughly speaking, when $q_{\perp}$ starts to approach the typical electron momentum $\left(\sim Z_{t}\right)$ in a free target ${ }^{2}$, and extends to very high (on the target scale) values of $q_{\perp}$. In this range the many-photon exchange should be taken into account. These two ranges are separated by the 'intermediate' range $Z_{t} \frac{Z_{t}}{v_{p}} \ll q_{\perp} \ll \lambda Z_{t}$ where the relativistic effects are already very weak and the many-photon exchange is still of minor importance. When $Z_{t}$ increases the 'intermediate' range tends to shrink. However, for not too high $Z_{t}$ this range is large enough, especially for relativistic collisions with very light targets, which are of a particular interest for the present study.

Thus, we are now in a position to formulate one of the interesting properties of the process of ionization of light targets by relativistic projectiles. If one restricts attention to emission of the main bulk of emitted electrons (electrons with not too high energy), then for the collision with a certain value of the momentum transfer either the relativistic effects or the many-photon exchange may be of importance. In other words, if the projectile-target interaction is transmitted by virtual photons with substantial 'transverse component' [14] then this interaction is well represented by the exchange of just a single photon. If, however, the many-photon exchange substantially contributes to the ionization process then it means that the exchanged photons are so-called 'longitudinal'.

4. These observations suggests to try to develop an approach using the following rather simple scheme. First, all collision events are divided into collisions with 'small' and 'large' momentum transfers. Second, collisions with 'small' momentum transfers which correspond to effectively large impact parameters, where the relativistic effects are important but the deviation from the perturbative behaviour is expected to be weak, are treated within the relativistic first order approximation. Third, collisions with 'large' momentum transfers, which in the case of light targets effectively occur due to the unretarded Coulomb interaction only, should be treated using an approach, which must account for the many-photon exchange between the projectile and the target but may still be purely nonrelativistic. Since we consider collisions with very high velocities, one can expect that an eikonal-like approximation would be very well suited to account for the many-photon exchange.

A crucial point for the approach outlined above is whether there exists an 'intermediate' range of momentum transfers where the first order relativistic treatment and a nonrelativistic eikonal approach yield close results. However, as the considerations of the subsections 1-3 suggest and as it will be also demonstrated for a particular case of $1 \mathrm{GeV} / \mathrm{U}^{92+}$-He collisions in the next section, such a condition can be fulfilled.

\footnotetext{
${ }^{2}$ The low boundary of this range depends also on the ratio $\frac{Z_{p}}{v_{p}}$, it increases with descreasing $\frac{Z_{p}}{v_{p}}$.
} 
5. As an eikonal-like approach, for treating collisions with 'large' momentum transfers, $q_{\perp} \gg q_{\min }$, we take the Glauber approximation [20]. This approximation has been widely used in high-energy, nuclear and atomic collision physics (for a review on some of its applications in atomic physics see [21], [22], see also [24] and references therein). It is very important to note that the eikonal form of Glauber can be derived from the formalism of quantum electrodynamics where it is obtained by summing all Feynmann graphs of the 'ladder' (or generalized 'ladder') type (see e.g. [25] and references therein) ${ }^{3}$.

In atomic physics the Glauber approximation is rather often employed even for collisions with such parameters where its application misses a clear physical justification but still yields rather good results. Since here we consider collisions with velocities approaching the speed of light, the conditions $\frac{Z_{p}}{v_{p}}<1$ and $v_{p} \gg v_{0}$ are fulfilled, where $v_{0} \sim Z_{t}$ is the typical orbiting velocity of the target electrons. The latter condition also means that typical velocities of the main bulk of the emitted electrons, $k \sim v_{0}$, are much smaller than the projectile velocity. The above conditions and the 'transverse' character of the collisions with $q_{\perp} \gg q_{\min }$ seem to provide a favourable situation for the Glauber approximation to be employed.

Within the Glauber approximation the cross section, differential in the momentum transfer $\mathbf{q}=\mathbf{P}_{i}-\mathbf{P}_{f}$, of a process where the target makes the transition $\psi_{0} \rightarrow \psi_{n}$ reads

$$
\frac{d \sigma_{0 \rightarrow n}}{d \mathbf{q}_{\perp}}=\frac{1}{2 \pi^{2}}\left|T_{n 0}^{G l}(\mathbf{q})\right|^{2}
$$

where

$$
T_{n 0}^{G l}(\mathbf{q})=i v_{p} \int d^{2} \mathbf{b} \exp (i \mathbf{q} \cdot \mathbf{b})\left\langle\psi_{n}\left|\prod_{j}\right| \mathbf{b}-\left.\boldsymbol{\rho}_{j}\right|^{-i \eta} b^{i \eta} \mid \psi_{0}\right\rangle
$$

Here $\eta=\frac{2 Z_{p}}{v_{p}}$ is the Sommerfeld parameter and $\boldsymbol{\rho}_{j}$ is the two-dimensional vector part of the $i$-th electron coordinate $\mathbf{r}_{j}$. The vector $\boldsymbol{\rho}_{j}$ lies in the plane defined by the vectors of the total momentum transfer $\mathbf{q}$ and the 'impact parameter' $\mathbf{b}$. As well known the choice of such a plane (more precisely, the choice of the corresponding eikonal path perpendicular to this plane) allows one to fully recover the (nonrelativistic) first-order Born amplitude as the first term of the $\eta$-expansion of the Glauber amplitude (5). The difference between the Glauber and first order transition amplitudes in collisions with 'large' momentum transfers accounts (approximately) for the many-photon exchange.

6. We note that the original version of the Glauber approximation [20], in which the longitudinal momentum transfer $q_{\min }$ is neglected, was recently applied to consider excitation of pionium in ultra-relativistic collisions with neutral atoms by Afanasyev et al [26] and Schumann et al [27]. Although these authors did not discuss the applicability of this approximation, which employs the unretarded interaction to treat ultra-relativistic collisions, it is quite clear that the justification of such a treatment is due to the fact that in collisions

\footnotetext{
${ }^{3}$ In the limit $v_{p} \rightarrow c$ this form can also be obtained by a direct solution of quantum mechanical equations for one particle in the electromagnetic field of the other particle which moves at the speed of light [19], [17].
} 
with neutral atoms only small impact parameters (or relatively large momentum transfers), where the relativistic effects are not important, contribute to excitation (and breakup) of pionium.

7. In the theory of ion-atom collisions the so-called two center effects (TCE) and the Coulomb boundary conditions $(\mathrm{CBC})$ have been attracting much attention during the last decades (see e.g. [28], [29] and references therein). The Glauber approximation can account for some of the two-center effects, e.g. for the angular asymmetry in the spectrum of emitted low-energy electrons which is due to the attraction of the emitted electrons by the outgoing projectile. Calculations in the Glauber approximation for the longitudinal spectrum of emitted electrons show a more pronounced forward-backward asymmetry in the spectrum compared to the first Born results. On the other hand, both the first order (in its usual form) and the Glauber approximations do not satisfy the CBC (in the Glauber transition amplitude only the initial state (in the post form) or only the final state (in the prior form) satisfies the CBC). Therefore, the approach of the present paper does not take into account the $\mathrm{CBC}$ and cannot describe such phenomena as e.g. the electron capture to the projectile continuum. However, as was already stated, in the present paper we consider ionization of light atoms in relativistic collisions where emitted electrons are nonrelativistic, i.e. they have velocities much smaller than that of the projectile. Thus, the present consideration is reduced to emission of soft electrons where the combination of the first order and Glauber ${ }^{4}$ approximations seems to be a reasonable and relatively simple tool.

8. To conclude this section we would like to note the following. In the semiclassical approximation a projectile and a target nucleus are regarded as moving along classical trajectories. Within this approximation collisions, in which the projectile-target interaction can be strong, are sometimes treated by dividing all impact parameters into 'small' and 'large' ones. Then the 'sudden-like' approximations and first order perturbation theories are applied to describe collisions with 'small' and 'large' impact parameters, respectively (see e.g. [30] and [31]). The proposed quantum approach, which deals with momentum transfers rather than with impact parameters, can be viewed as complementary to the above mentioned semiclassical treatments.

\section{RESULTS AND DISCUSSION}

In relativistic collisions between highly charged ions and light targets very substantial differences between results of the first order treatment and of that, which also includes contributions from the higher order terms in the corresponding Born series in the projectiletarget interaction, are first of all expected for processes in which at least two target electrons

\footnotetext{
${ }^{4}$ It is worth to mention that one may face substantial difficulties in the generalization of nonrelativistic multiple-scattering theories, where kinetic energy terms stand for the perturbation in the transition amplitude such as e.g. the symmetrized eikonal approximation, for the domain of relativistic collision energies (see discussion in [2]).
} 
actively participate. However, it is instructive to begin the study of the nonperturbative behaviour in relativistic collisions by considering how the influence of the higher order terms might change differential and total cross sections for target single ionization, a process where this influence is expected to be, on average, rather weak [16].

From the theoretical point of view it is tempting to apply the proposed approach for collisions of relativistic highly charged projectiles with atomic hydrogen target where exact target states are known. However, there is no experimental data on hydrogen ionization (or excitation) in such collisions. The simplest target, for which such experimental data are available, is helium. Therefore, in the rest of this section we restrict our attention to considering some aspects of helium single ionization by relativistic highly charged ions.

Results of the only kinematically complete experiment on atom ionization by a projectile in the relativistic domain of collision velocities were presented in [9]. In this experiment measurements were performed for the spectrum of electrons emitted in the process of helium single ionization by $1 \mathrm{GeV} / \mathrm{u} \mathrm{U}^{92+}$ projectiles. The collision energy corresponds to a collision velocity of $120 \mathrm{a} . \mathrm{u}$. and a collision Lorentz factor of 2.07. The detailed theoretical study of this process, based on the relativistic Born approximation, was recently reported in [16].

If one considers collisions with atomic targets, which have more than one electron, then, in addition to the problem of dealing with the projectile-target interaction, the question of describing target states arises ${ }^{5}$. In our calculations which we report here the same option as in [16] was used to describe the initial and final states of the helium atom. The wavefunctions

$$
\psi_{i}\left(\mathbf{r}_{1}, \mathbf{r}_{2}\right)=N\left(e^{-a r_{1}-b r_{2}}+e^{-b r_{1}-a r_{2}}\right),
$$

with $N=0.709 a=1.18853$ and $b=2.18317$, and

$$
\psi_{f}\left(\mathbf{r}_{1}, \mathbf{r}_{2}\right)=\frac{1}{\sqrt{2}}\left(\phi_{\mathbf{k}}\left(\mathbf{r}_{1}, Z=1\right) \phi_{0}\left(\mathbf{r}_{2}, Z=2\right)+\phi_{\mathbf{k}}\left(\mathbf{r}_{2}, Z=1\right) \phi_{0}\left(\mathbf{r}_{1}, Z=2\right)\right)
$$

were taken as the initial and final states of helium, respectively. In (7) the state $\phi_{0}(Z=2)$ is the ground state of $\mathrm{He}^{+}$and $\phi_{\mathbf{k}}(Z=1)$ is the Coulomb continuum state in the field of a charge $Z=1$ with $\mathbf{k}$ being the momentum of the emitted electron. Similarly to [16] (see also [23]), the bound and continuum states were orthogonalized by using the Schmidt procedure $\psi_{f} \rightarrow \psi_{f}-<\psi_{i} \mid \psi_{f}>\psi_{i}$.

Often, if the projectile-target interaction is treated beyond the first order approach, the electron dynamics in a multi-electron target is considered within the one (active) electron approximation, i.e. is basically reduced to the single-electron dynamics. The simplest approximation is to assume that the electron moves in an hydrogen-like Coulomb field with some effective charge, which in the case of helium single ionization is often taken as $Z_{\text {eff }}=1.345$ (see [32], [28] and references therein). More sophisticated descriptions involve the application of Clementi-Roetti wavefunctions and the use of initial and final states calculated with

\footnotetext{
${ }^{5}$ Of course, different options for approximating target states will yield, in general, different results for cross sections. This is, however, a separate problem which is not connected to the point whether all collisions can be subdivided into those with the 'small', 'intermediate' and 'large' momentum transfers, i.e. to the point whether the proposed approach to treat the projectile-target interaction can be applied.
} 
a Hartree-Fock potential. Such (free) target descriptions were combined with the CDWCDW [33] and CDW-EIS [34] approximations in [35]. We also note that in the case of electron detachment from a negative hydrogen ion the explicit four-body formulation with using two-electron wavefunctions was considered in [36] (see also [29]).

Concerning our case, the choice of the states (6) and (7), although rather simple, represents the first attempt to explicitly address, beyond the first order approximation, the four-particle dynamics in collisions with highly charged ions in the domain of very high (relativistic) collision velocities.

\section{Fully differential cross section.}

Let us first consider the fully differential cross section. In figure 1 we display results of calculations for the cross section performed within the first order approximation and the approach discussed in the previous section. The transverse momentum transfer and the electron emission energy are taken to be $q_{\perp}=1$ a.u. and $\varepsilon_{k}=20 \mathrm{eV}$, respectively. The present value of the momentum transfer is quite large, so at the chosen values of $Z_{p}=92$ and $v_{p}=120$ we are in the region of the momentum transfer, where substantial deviations from the first order consideration can be expected but the relativistic effects are already negligible.

In figure $1 \mathrm{a}$ the cross section is shown as a function of the polar emission angle, $\theta_{k}=$ $\arccos \left(\left(\mathbf{k} \cdot \mathbf{v}_{p}\right) /\left(k v_{p}\right)\right)$, for the case of coplanar geometry $\left(\varphi_{k}=0^{0}\right.$, the azimuthal emission angle $\varphi_{k}$ is counted from the direction of $\mathbf{q}_{\perp}$ ). In this case both, the first order and eikonal calculations, show a rather similar shape of the cross section but result in considerably different absolute values. The fact that the first order approach yields in this case larger results corresponds to the rather general tendency observed when the many-photon exchange is taken into account. Namely, the inclusion of the higher order terms in the projectile-target interaction reduces calculated values of the total ionization cross section compared to those values which follow from first order approaches (see also figure 3 ).

A more interesting manifestation of the many-photon exchange is observed in calculations if one looks at the cross section in the plane where the azimuthal emission angle $\varphi_{k}=90^{0}$. For this geometry the fully differential cross section is displayed in figure $1 \mathrm{~b}$ for the same values of $Z_{p}, v_{p}, q_{\perp}$ and $\varepsilon_{k}$. While the first order calculation suggests quite a flat dependence on the angle $\theta_{k}$, the Glauber approximation predicts for this dependence a double-maxima structure with maxima centered at $\theta_{k}=90^{\circ}$ and $270^{\circ}$. The origin of the flat dependence on $\theta_{k}$ in the first order calculation is quite transparent since within the first order approach the emission geometry is basically determined only by the vector of the momentum transfer $\mathbf{q}$ which, in the case under consideration, is nearly equal to its transverse part $\mathbf{q}_{\perp}$ and the cross section should be nearly a constant in the plane perpendicular to $\mathbf{q}_{\perp}$. The formal reason for the difference between the eikonal and the first order results is that within the eikonal approach two vectors, the momentum transfer $\mathbf{q} \approx \mathbf{q}_{\perp}$ and the projectile velocity $\mathbf{v}_{p}$, determine the general emission geometry. Since for the collision parameters in question the Glauber approximation seems to have an advantage over the first order approach we believe that the former should yield results for the cross section which are closer to reality. ${ }^{6}$

\footnotetext{
${ }^{6}$ In the experiment [9] the fully differential cross sections were not extracted because of statistics which was not good enough. However, a similar two-maxima structure (but with substantially
} 
We, however, were not able to draw any simple physical picture explaining the appearance of the two maxima in the eikonal calculation.

Before turning to the discussion of electron emission spectra we illustrate in figures 2 and 3 the existence of the 'intermediate' range of the transverse momentum transfers for the present collision system. In figure 2 we plot the fully differential cross section in the coplanar geometry for $q_{\perp}=0.2$ a.u. and $\varepsilon_{k}=20 \mathrm{eV}$. It is seen that the relativistic first order and the nonrelativistic eikonal approximations yield close results for the cross section. In figure 3 the ionization cross section differential in $q_{\perp}$ is shown. This cross section was obtained by integrating over the emitted electrons with energies $0 \leq \varepsilon_{k} \leq 400 \mathrm{eV}$. It follows from the calculation (see also figure 3) that both the relativistic first order and the eikonal theories yield very close results for the projectile scattering in the ionizing collisions with $0.07 \leq q_{\perp} \leq 0.3$ a.u.. With increasing $q_{\perp}$ the contribution to the cross section rapidly decreases. We note, however, that according to figure 3 there exists the range of not too large momentum transfers $q_{\perp}$, which still noticeably contributes to the total cross section, where the first order theory gives substantially larger results. The latter reflects the rather general fact that the first order theories may overestimate cross sections in the case of strong perturbations and is consistent with the relation between the eikonal and first order results which are shown in figure 1.

\section{Evaluation of cross sections integrated over the momentum transfer.}

Within the present approach cross sections differential only in electron variables were calculated as follows. According to the discussion in the previous section, all collisions were subdivided into collisions with small, $0 \leq q_{\perp} \leq q_{\perp}^{0}$, and 'large', $q_{\perp}>q_{\perp}^{0}$, momentum transfers. Collisions with $q_{\perp} \leq q_{\perp}^{0}$ were treated in the relativistic first order approximation. Collisions with $q_{\perp}>q_{\perp}^{0}$ were considered in the Glauber approximation. The value of $q_{\perp}^{0}$ was taken dependent on the energy of the emitted electron (e.g. from $q_{\perp}^{0}=0.12$ a.u. for electrons with $\varepsilon_{k} \leq 1$ a.u. up to $q_{\perp}^{0}=0.25$ a.u. for electrons with $3 \leq \varepsilon_{k} \leq 4$ a.u.). It turned

out that the result was not sensitive to a particular choice of $q_{\perp}^{0}$ provided $0.1 \lesssim q_{\perp}^{0} \lesssim 0.3$ a.u.. The latter is important and lends an additional support to the existence of the 'intermediate' range of $q_{\perp}$ where both the (relativistic) first order and (nonrelativistic) Glauber approximations yield quite close results.

\section{Cross sections differential in the electron emission energy and polar angle.}

broader maxima) in the fully differential cross section in the plane $\varphi_{k}=90^{0}$ was recently observed experimentally [37] in helium single ionization by $100 \mathrm{MeV} / \mathrm{u} \mathrm{C}^{6+}$ projectiles at $q_{\perp} \sim 1$. Calculations with the states (6) and (7) for this collision system, where the effective perturbation strength $Z_{p} / v_{p} \approx 0.1$ is much smaller compared to that in collisions with $1 \mathrm{GeV} / \mathrm{u} \mathrm{U}^{92+}\left(Z_{p} / v_{p}=0.77\right)$, suggest that such a structure might exist at $q_{\perp} \gtrsim 1$. One should mention that in the case of weak perturbation $\left(Z_{p} / v_{p} \ll 1\right)$ the results in the plane $\varphi_{k}=90^{0}$ turned out to be very sensitive to the choice of the target states. The latter, however, is not the case for strong perturbations $\left(Z_{p} / v_{p} \lesssim 1\right.$, like e.g. in collisions with $1 \mathrm{Gev} / \mathrm{u} \mathrm{U}^{92+}$ ) where the application of different target states yields rather similar results. 
The double differential cross section $\frac{d^{2} \sigma^{+}}{d \varepsilon_{k} \sin \theta_{k} d \theta_{k}}$ is displayed in figure 4 as a function of the polar emission angle $\theta_{k}$ for fixed electron emission energies of 0.1 and 1 a.u., respectively. As in the previous subsection we consider the reaction $1 \mathrm{GeV} / \mathrm{u} \mathrm{U}^{92+}+\mathrm{He} \rightarrow \mathrm{U}^{92+}+\mathrm{He}^{+}+$ $\mathrm{e}^{-}$. Ionization cross section differential in the polar angle of the emitted electron, $\frac{d \sigma^{+}}{\sin \theta_{k} d \theta_{k}}$, is shown in figure 5. In both figures results of the first order calculations are represented by dashed curves whereas results of the present approach, which combines the first order and Glauber approximations, are given by solid curves.

It is intuitively clear and is also suggested by earlier considerations [12] that at this very high collision velocity, when $v_{p} \gg v_{0}$ and $Z_{p} / v_{p}^{2} \ll 1$, the emission of electrons with $k \sim v_{0}$ proceeds nearly symmetrically with respect to the angle $\theta_{k}=90^{\circ}$. The same conclusion follows from both the present calculations displayed in figures 4 and 5. According to the latters, the main difference between the first order and present approaches is that the present approach yields considerably smaller results for the emission at angles close to $\pi / 2$ where the cross sections reach their maximal values. For the angles close to 0 and $\pi$, where the corresponding cross sections are rather small, the difference between them decreases.

Two main conclusions can be draw from the study of the doubly and singly differential cross sections. First, at the collision parameters considered the account of the many-photon exchange do not influence much the angular distribution of the electron emission compared to that following from the first order calculation. Second, even at this very high velocity the account of the many-photon exchange results in considerably smaller values of the total emission. These two points can be understood by noting that the contribution to the angular asymmetry, which may arise from the post-collision interaction is, roughly speaking, proportional to $Z_{p}^{3} /\left(v_{p}^{4} \gamma^{2}\right)$ (see [12]) and for $v_{p}=120$ and $\gamma=2.07$ is very small even at $Z_{p}=92$. At the same time, the non-first order effects in the total cross section are determined by the factor $Z_{p} / v_{p}$ (see e.g. [30]), which in the case under consideration is not very far from unity.

\section{Electron energy spectrum.}

The energy spectrum of the emitted electrons is displayed in figure 6 . It is seen in figure $6 \mathrm{a}, \mathrm{b}$ that the difference between the two calculations is just about $10 \%$ at lower electron energies $\left(\varepsilon_{k} \sim 0-5 \mathrm{eV}\right)$. However, the difference increases with increasing emission energy and reaches already about $40 \%$ at $\varepsilon_{k} \approx 100 \mathrm{eV}$. The inclusion of the many-photon exchange reduces the calculated total cross section for helium single ionization by approximately $20 \%$, somewhat larger than we expected based on estimates for the total cross section obtained in $[30]$.

It is seen from figure $2 \mathrm{c}, \mathrm{d}$ that both, the first order and the proposed approaches, yield results which are in reasonably good agreement with the experimental data. It seems, however, that the latter approach improves the agreement for the higher-energy part of the electron spectrum.

\section{Distributions of longitudinal momenta of emitted electrons.}

The fact, that the proposed approach slightly improves agreement with experiment, can also be observed in figure 7 . In this figure we show results of calculations for the electron emission spectrum plotted as a function of the longitudinal component, $k_{\text {long }}$, of the momentum of the emitted electron. The minimal difference between the first order result and that of the 
present approach takes place at $k_{\text {long }}=0$ a.u. $(\approx 15 \%)$. When the absolute value of $k_{\text {long }}$ increases the difference also increase and reaches about $35-40 \%$ at $k_{\text {long }}= \pm 2.5$ a.u.. It is seen from the figure 7 that the account of the many-photon exchange brings the calculated results in a closer agreement with the experimental data.

\section{CONCLUSION}

We have presented an approach for describing ionization of light atoms in relativistic collisions with highly charged ions. This approach consists of dividing all collisions into those with 'small' and 'large' momentum transfers. The former and the latter are described by the first order relativistic theory and the Glauber approximation, respectively. Such an approach allows one to account (approximately) for many-photon exchanges. The present approach can be regarded as complementary to those semiclassical treatments where collisions with 'small' and 'large' impact parameters are described by the 'sudden'-like and first order approximations, respectively.

As the first application of this approach, it was used to describe helium single ionization by $1 \mathrm{GeV} / \mathrm{u} \mathrm{U}^{92+}$ projectiles. In this case a striking difference with the first order results is obtained only for the fully differential cross section and only when the transverse momentum transfer $q_{\perp}$ is relatively large. For the electron emission cross sections, integrated over the momentum transfer, the differences between results of the first order and the proposed approaches are, on average, relatively small. In particular, they turned out to be quite substantial only for the higher energy part of the emitted electron spectra which is of minor importance for the total emission. It has been demonstrated, however, that, provided the same initial and final states of helium (6) and (7) are employed in both the first order and the proposed approaches, the latter allows one to bring calculated results for the emission cross sections to a better agreement with available experimental data.

Much more pronounced manifestations of the many-photon exchange between colliding projectile and target are expected in the process of helium double ionization by relativistic highly charged ions. Note that there exist experimental data on spectra of electrons emitted in the process of helium double ionization by $1 \mathrm{GeV} / \mathrm{u} \mathrm{U}^{92+}$ projectiles [9], [10]. The work on this topic is in progress.

\section{ACKNOWLEDGEMENTS}

The authors acknowledge with thanks the support from the Deutsche Forschungsgemeinschaft (DFG), from the Max-Planck-Institut für Kernphysik and the Leibniz-Program of the DFG. 


\section{REFERENCES}

[1] J. Eichler and W.E. Meyerhof Relativistic Atomic Collisions (Academic Press, San Diego, 1995 )

[2] J. Eichler, Phys. Rep. 193165 (1990)

[3] C.A. Bertulani and G. Baur, Phys. Rep. 163299 (1988)

[4] R. Anholt and H. Gould, Adv. At. Mol. Phys. 22315 (1986)

[5] H.Berg et al, Phys.Rev. A 465539 (1992)

[6] J. Ullrich et al, Phys.Rev.Lett. 711697 (1993)

[7] S.Hofstetter, N.Grün and W.Scheid, Z.Phys. 371 (1996)

[8] S.Keller, H.J.Lüdde and R.M.Dreizler, Phys.Rev. A 554215 (1997)

[9] R.Moshammer et al, Phys.Rev.Lett. 793621 (1997)

[10] C.J.Wood, R.E.Olson, W.Schmidt, R.Moshammer, and J.Ullrich, Phys.Rev. A56 3746 (1997)

[11] C.Pfeiffer, N.Grün and W.Scheid, J.Phys. B 3253 (1999)

[12] A.B.Voitkiv, N.Grün and W.Scheid, J.Phys. B32 101 (1999), 3923 (1999)

[13] J. Ullrich, H. Schmidt-Böcking, S. Kelbch, H. Berg, S. Hagmann, P. Richard, A.S. Schlachter and R. Mann, NIM B 23131 (1987)

[14] A.B. Voitkiv and J. Ullrich, J. Phys. B34 4513 (2001)

[15] B. Najjari, A.B. Voitkiv and J. Ullrich, J. Phys. B 35533 (2002)

[16] A.B. Voitkiv. B. Najjari, R. Moshammer and J. Ullrich, Phys. Rev. A 65032707 (2002)

[17] A. J. Baltz, Phys.Rev.Lett. 781231 (1997)

[18] G. t'Hooft, Phys. Lett. B 19861 (1987)

[19] R. Jakiw, D. Kabat and M. Ortiz, Phys. Lett. B 277148 (1992)

[20] R.J. Glauber, in Lectures in Theoretical Physics 315 (edited by W.E.Brittin and L.G.Dunham, Interscience Publishers, New York, 1959)

[21] E. Gerjuoy and B.K. Thomas, Rep.Progr.Phys. 371346 (1974)

[22] C.J. Joachain and C. Quigg, Rev.Mod.Phys. 46279 (1974)

[23] F.W.Byron Jr, C.J.Joachain and B.Piraux, J.Phys. B 191201 (1986); H.Fukuda, I.Shimamura, L.Vegh, and T.Watanabe, Phys.Rev. 441565 (1991)

[24] J.H. McGuire, Phys.Rev. A 26143 (1982)

[25] H.D.I Abarbanel and C. Itzykson, Phys.Rev.Lett. 231 (1969); M. Levy and J. Sucher, Phys.Rev. 1861656 (1969); H. Fried, Functional methods and models in quantum field theory (MIT press, Cambridge, MA, 1972)

[26] L.G. Afanasyev, A. Tarasov and O. Voskresenskaya, J.Phys. G 25 B7 (1999)

[27] M. Schumann, T. Heim, D. Trautmann and G. Baur, J.Phys. B 352683 (2002)

[28] N.Stolterfoht, R.D.DuBois and R.D.Rivarola, Electron Emission in Heavy Ion-Atom Collisions (Springer, Berlin, Heidelberg, 1997)

[29] Dz. Belkic, J. Comp. Meth. Sci. Eng. 11 (2001)

[30] A.B.Voitkiv and A.V.Koval', J.Phys. B 31398 (1998)

[31] A.J. Baltz, Phys. Rev. A 61042701 (2000), A.B.Voitkiv, C.Müller and N.Grün, Phys. Rev. A 62062701 (2000)

[32] J.H. McGuire, A. Mueller, B. Schuch, W. Groh and E. Salzborn, Phys. Rev. A 353265 (1987); R. Anholt, W.E. Meyerhof, X.-Y. Xu, H. Gould, B. Feinberg, R.J. McDonald, H.E. Wegner and P. Thieberger, Phys.Rev. A 361586 (1997); A. Igarashi, N. Toshima, and T. Ishihara, Phys. Rev. A 45 R6157 (1992) 
[33] Dz. Belkic, J.Phys. B 113529 (1978)

[34] D.S.F.Crothers and J.F.McCann, J.Phys. B 163229 (1983)

[35] P.D.Fainstein, V.H.Ponce and R.D.Rivarola, J.Phys. B 221207 (1989); P.D.Fainstein et al, J.Phys. B 27 L259 (1994); L.Gulyas, P.D.Fainstein and A.Salin, J.Phys. B 28245 (1995); S.F.C.O'Rourke, I.Shimamura and D.S.F.Crothers, Proc.R.Soc.Lond. A 452 175 (1996); S.F.C.O'Rourke and D.S.F.Crothers, J.Phys. B 302443 (1997); L.Gulyas and P.D.Fainstein, J.Phys. B 313297 (1998) ; T. Kirchner, L. Gulyas, R. Moshammer, M. Schulz and J. Ullrich, Phys. Rev. A 65042727 (2002)

[36] Dz. Belkic, J.Phys. B 301731 (1997)

[37] R.Moshammer, private communication. 
FIGURE CAPTIONS 
FIGURES

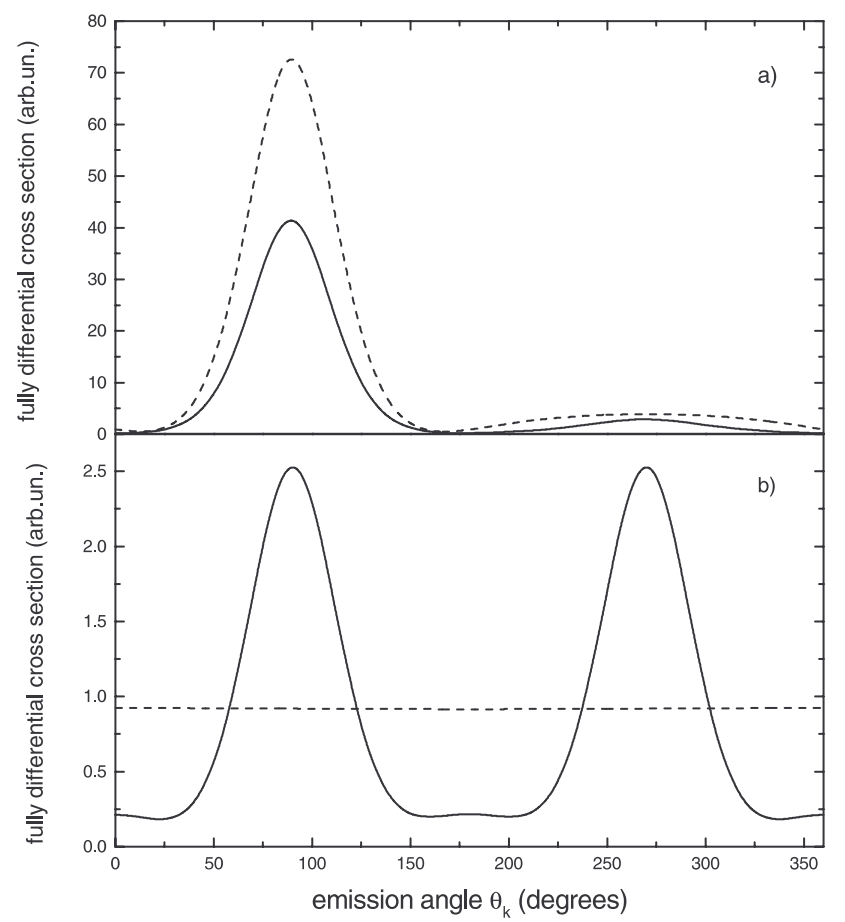

Figure 1

FIG. 1. Helium single ionization by $1 \mathrm{GeV} / \mathrm{u} \mathrm{U}^{92+}$ projectiles. The fully differential cross section $\frac{d \sigma^{+}}{d \varepsilon_{k} d \Omega_{k} d^{2} \mathbf{q}_{\perp}}$ as a function of the polar emission angle $\theta_{k}$ for a fixed emission energy $\varepsilon_{k}=20$ $\mathrm{eV}$ and transverse momentum transfer $q_{\perp}=1$ a.u.. a) The case of coplanar geometry, $\varphi_{k}=0^{0}$. b) The case of $\varphi_{k}=90^{\circ}$. Full curves: result of the eikonal approximation; dashed curves: result of the relativistic first Born approximation. 


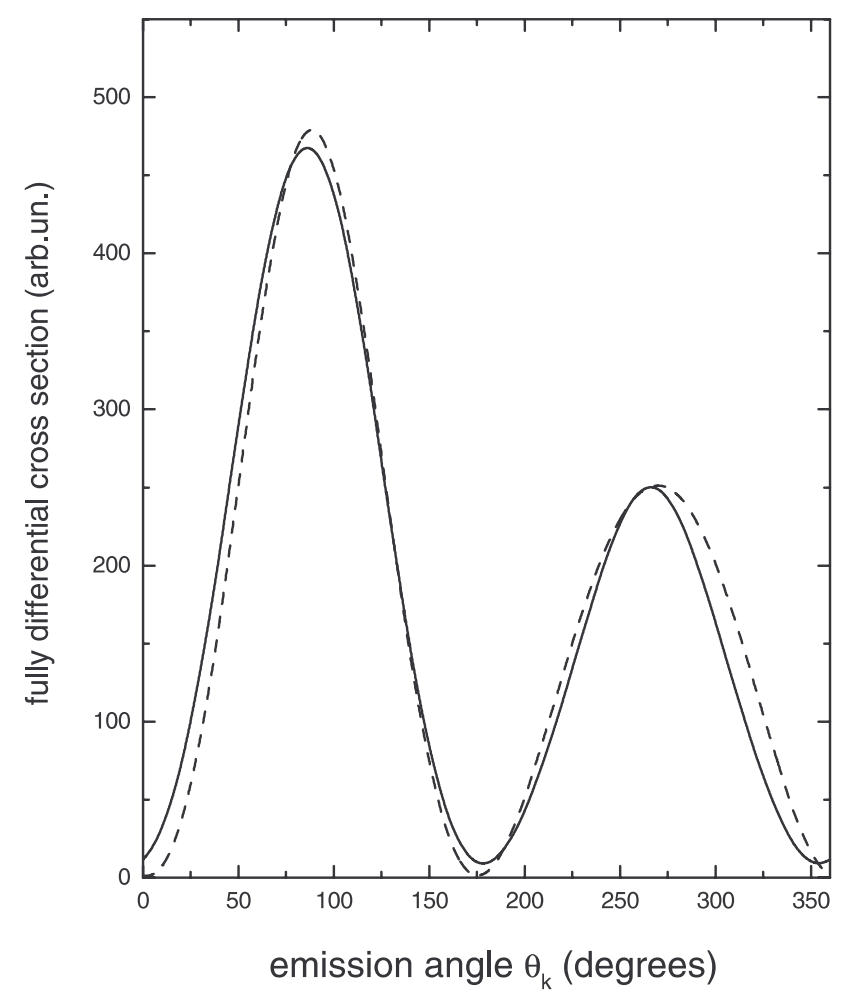

Figure 2

FIG. 2. Same as in figure 1a but for $q_{\perp}=0.2$ a.u.. 




\section{Figure 3}

FIG. 3. Helium single ionization by $1 \mathrm{GeV} / \mathrm{u} \mathrm{U}^{92+}$ projectiles. Singly differential cross section $\frac{d \sigma^{+}}{d q_{\perp}}$ is obtained by integrating over electrons emitted with energies $0 \leq \varepsilon_{k} \leq 400 \mathrm{eV}$. Full curve: result of the eikonal approximation; dashed curve: result of the relativistic first Born approximation. (The range of the larger momentum transfers, $q_{\perp}>4$ a.u., is not shown since in this range the higher emission energies, $\varepsilon_{k}>400 \mathrm{eV}$, start to noticeably contribute to the cross section.) 


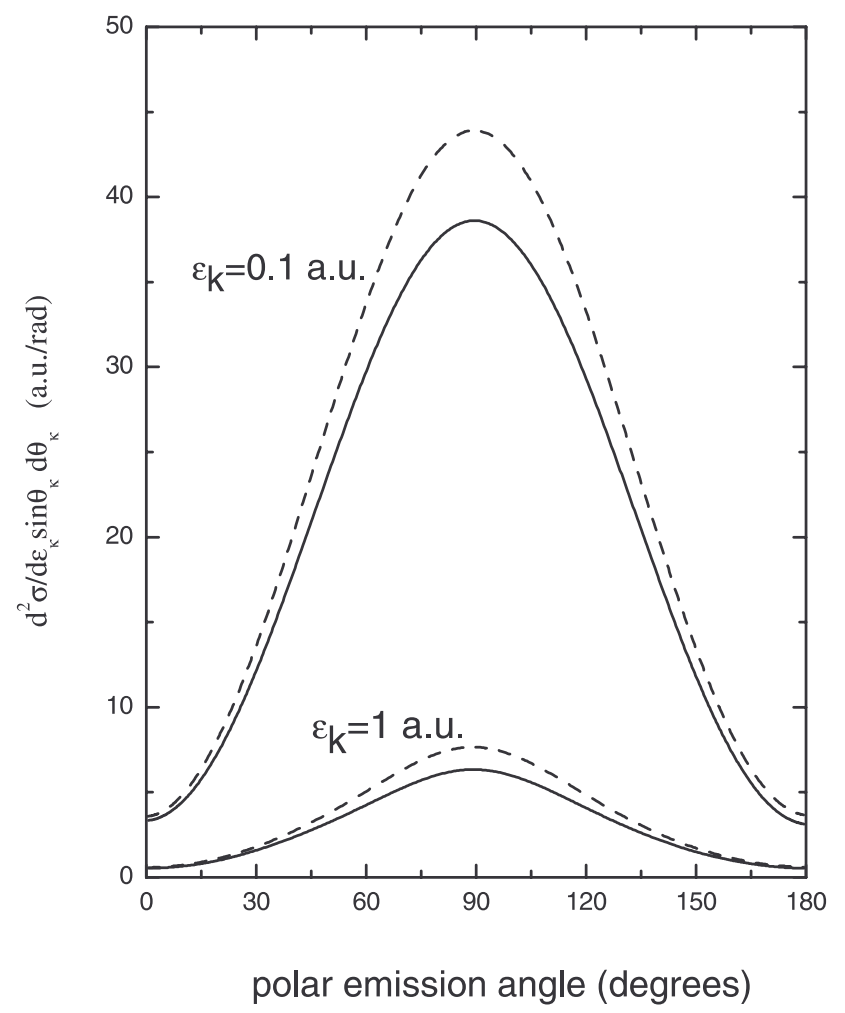

Figure 4

FIG. 4. Helium single ionization by $1 \mathrm{GeV} / \mathrm{u} \mathrm{U}^{92+}$ projectiles. Doubly differential cross section $\frac{d^{2} \sigma^{+}}{d \varepsilon_{k} \sin \theta_{k} d \theta_{k}}$ as a function of the polar emission angle at fixed emission energies of 0.1 and 1 a.u.. Dashed curves: results of the relativistic first Born approximation; solid curves: results of the proposed approach. 


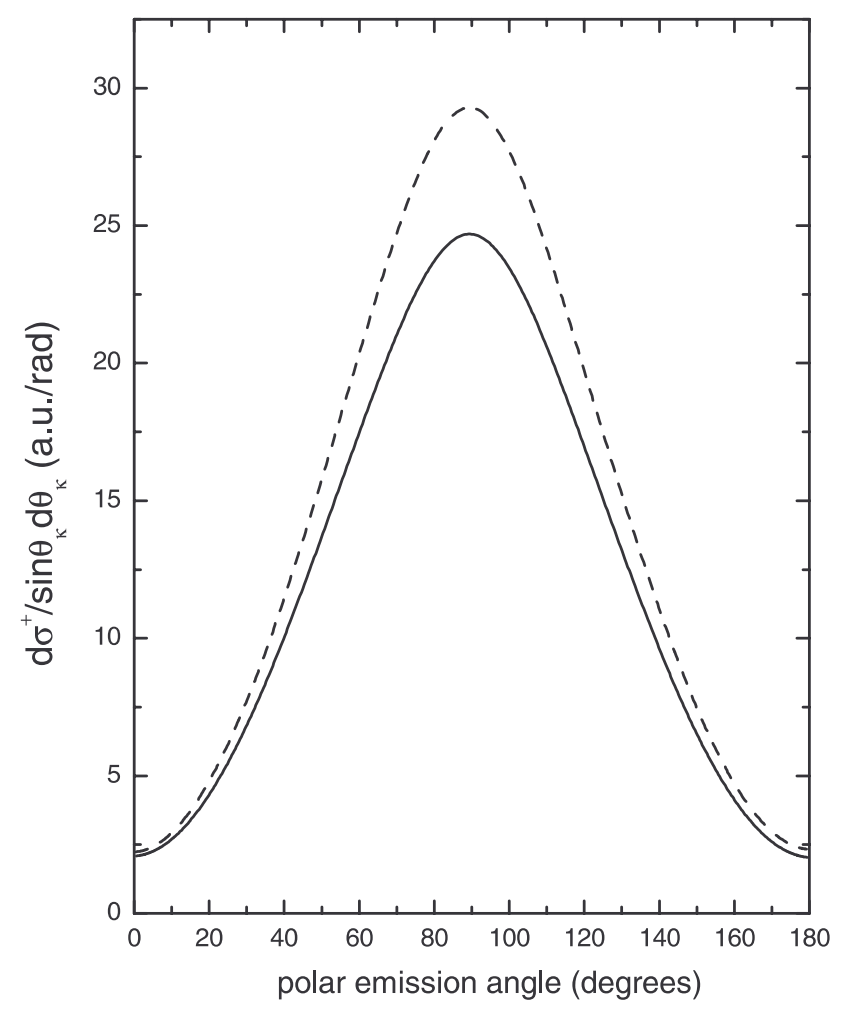

\section{Figure 5}

FIG. 5. Helium single ionization by $1 \mathrm{GeV} / \mathrm{u} \mathrm{U}^{92+}$ projectiles. Singly differential cross section $\frac{d \sigma^{+}}{\sin \theta_{k} d \theta_{k}}$ was obtained by integrating over electrons emitted with energies $0 \leq \varepsilon_{k} \leq 400 \mathrm{eV}$. Full curve: result of the proposed approach; dashed curve: result of the relativistic first Born approximation. 



Figure 6

FIG. 6. Energy distribution of electrons emitted in the process of helium single ionization by $1 \mathrm{GeV} / \mathrm{u} \mathrm{U}^{92+}$ impact. a) Dashed curve: result of the relativistic first Born approximation; solid curve: result of the proposed approach. b) The ratio of the cross sections shown in figure 6a. c) Circles: experimental results [9]; dashed curve: identical to dashed curve in a). d) Circles: experimental results [9]; solid curve: identical to solid curve in a). The experimental results shown in c) and d) have been normalized to the corresponding calculated results (more precisely, to the lower energy part of these results, $\varepsilon_{k} \lesssim 24.6 \mathrm{eV}$, which gives the main contribution to the emission). 


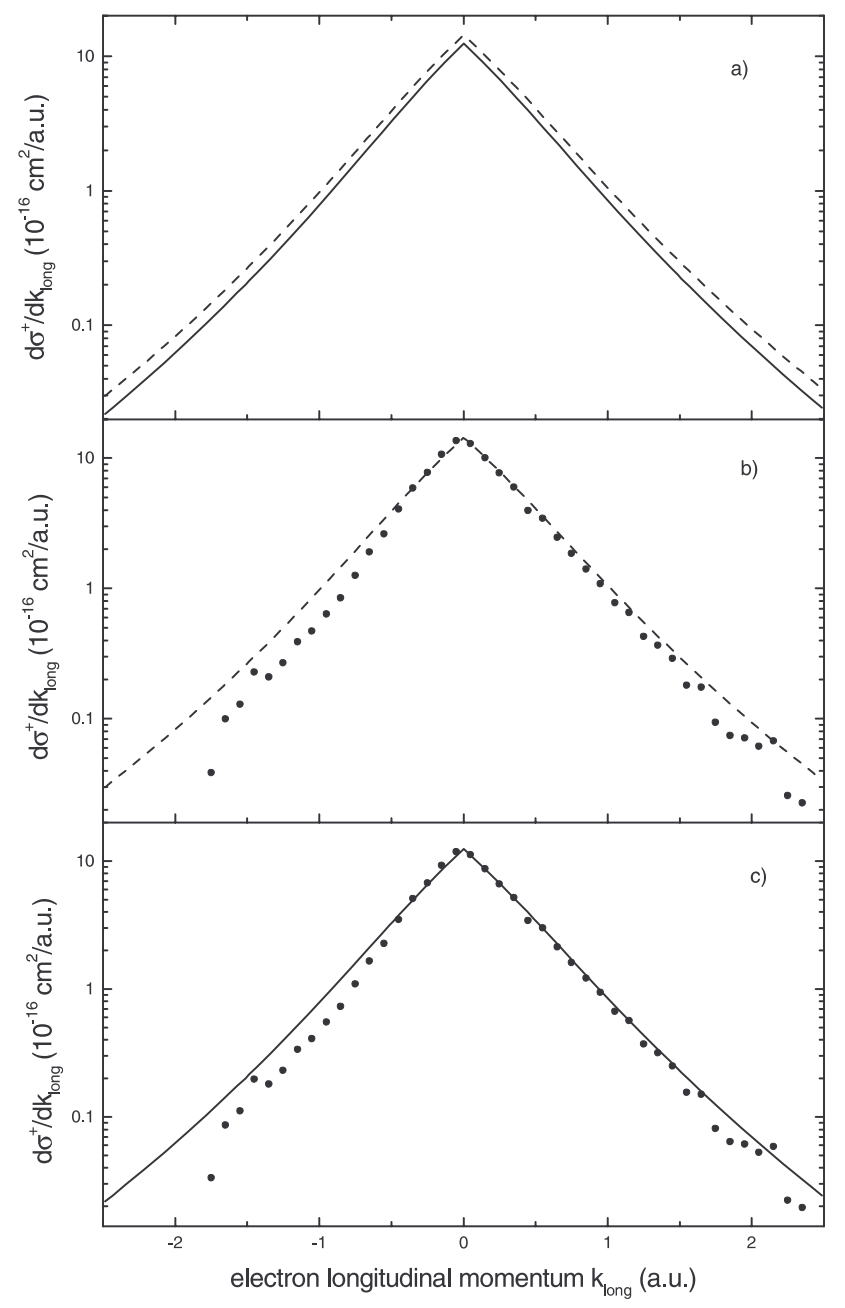

Figure 7

FIG. 7. Longitudinal momentum distribution of electrons emitted in the process of helium single ionization by $1 \mathrm{GeV} / \mathrm{u} \mathrm{U}^{92+}$ impact. a) Dashed curve: result of the relativistic first Born approximation; solid curve: result of the proposed approach. b) Circles: experimental results [9]; dashed curve: identical to dashed curve in a). c) Circles: experimental results [9]; solid curve: identical to solid curve in a). The experimental results shown in b) and c) were normalized to the corresponding calculated results. 